\title{
Impact of Interference on the Performance of Selection Based Parallel Multiuser Scheduling
}

\author{
Sung Sik Nam ${ }^{\dagger}$, Mohamed-Slim Alouini ${ }^{\ddagger}$ and Hong-Chuan Yang ${ }^{\dagger \dagger}$ \\ $\dagger$ Dept. of ECE, TAMU \\ College Station, Texas \\ Email: ssnam11@tamu.edu \\ $\dagger \dagger$ Dept. of ECE, Univ. of Victoria, \\ Victoria, Canada \\ Email: hyang@ece.uvic.ca
}

\begin{abstract}
In this paper, we investigate the impact of interference on the performance of selection based parallel multiuser scheduling. In conventional parallel multiuser scheduling schemes, every scheduled user is interfering with every other scheduled user. This is a factor which limits the capacity and performance of multiuser systems and the level of interference becomes substantial as the number of scheduled users increases. Based on the above motivation, we investigate the trade-off between the throughput and the number of scheduled users. The major difficulty in the total average sum rate capacity and the average spectral efficiency (ASE) analysis resides in the determination of the statistics of the Signal to Interference plus Noise Ration (SINR) of the scheduled user. Fortunately, we could derive the accurate statistical characterization of the SINR of the scheduled user. With these accurate statistical analysis, we derive the total average sum rate capacity and the average spectral efficiency based on the SINR. Based on the ASE and the total average sum rate capacity analysis, we investigate the trade-off between the throughput and the number of scheduled users. Hence, based on these results, the system should carefully select the appropriate number of scheduled users to maximize the overall throughput while maintaining an acceptable quality of service given certain channel conditions.
\end{abstract}

\section{Introduction}

Research in multiuser scheduling schemes for single users [1-3] and for multiple users [4,5] has grown in recent years. Single user scheduling schemes [1-3] schedule only one user at a time. On the other hand, parallel multiuser scheduling schemes [4,5] schedule multiple users at a time. The goal is to increase the throughput and to reduce the complexity of implementation.

This work was supported by Qatar Telecom (Qtel), Qatar.
The throughput of multiuser scheduling schemes for single user [1-3] and multiple users [4,5] was investigated assuming that the users are scheduled based on their signal to noise ration (SNR).

In conventional parallel multiuser scheduling schemes, a particular scheduled user's signal is detected by correlating signals of entired scheduled users at the receiver. Therefore, under these practical conditions, every scheduled user is interfering with every other scheduled user. These effect is called Multiple Access Interference (MAI) or Multiple User Interference (MUI). This MUI is a factor which limits the capacity and performance of multiuser systems and the level of interference becomes substantial as the number of scheduled users increases. To take into account the effect of MUI caused by the other scheduled users, we use in this paper the signal to interference plus noise ratio (SINR) which measures the ratio between the useful power and the amount of noise and interferences generated by all the other scheduled users. The level of interference becomes substantial as the number of the scheduled users increases because the SINR can decrease considerably in these conditions. This decrease in SINR can lead to a certain reduction of the rate allocated to each scheduled user.

With the above motivation in mind, we investigate in this paper the impact of interference on the performance (throughput) of the scheduled users assuming a selection based parallel multiuser scheduling scheme [4]. In particular, we derive the total average sum rate capacity and the average spectral efficiency (ASE) based on the SINR of the scheduled users. The major difficulty in deriving this total average sum rate capacity and ASE resides in the determination of the statistics of the SINR of the $m$-th scheduled user. Fortunately, extending the approach used in $[6,7]$ to derive some useful order statistics of interest, we accurately characterize the statistics of the SINR of the $m$-th scheduled user. These results are then applied to obtain the total average sum rate capacity and the 
ASE.

\section{System and Channel Models}

In our system model, we consider a code division multiple access (CDMA) system for the simultaneous scheduling of $K_{s}\left(K_{s}=0,1,2, \ldots, K\right)$ users among $K$ potential users per time-slot. We assume that there is some inter-user interference after the scheduling process. We also assume that the schemes have a reliable feedback path between the receiver and transmitter and that they are implemented in a discrete-time fashion with a time-slot composed of a guard time period followed by a data transmitting time period. During the guard time period, the system makes the necessary actions for scheduling the best $K_{s}$ users based on SNR information then estimating the SINRs of these scheduled users for rate allocation. Finally, it is assumed that the channel estimation is perfect at the receiver and that the feedback to the transmitter is performed upon request without any error.

We denote by $\gamma_{i}(i=1,2, \ldots, K)$, the received SNR of the $i$ th user and we adopt a block flat fading channel model. More specifically, assuming slowly-varying fading conditions, the different paths from users experience roughly the same fading conditions during the data burst and its preceding guard time period. In addition, the fading conditions are assumed to be independent across the paths from users and between guard time period and data burst pairs.

Based on the mode of operation of the proposed selection based parallel multiuser scheduling scheme, we select the best $K_{s}$ users for scheduling among the $K$ users. Therefore, if we consider a general communication system and we let $\gamma_{m: K}$ be a SNR of $m$-th scheduled user $\left(m=1,2, \ldots, K_{s}\right)$ then the SINR of this user can be written as

$$
S I N R_{m}=\gamma_{S I N R_{m}}=\frac{\gamma_{m: K}}{1+\alpha \sum_{\substack{n=1 \\ n \neq m}}^{K_{s}} \gamma_{n: K}},
$$

where $\alpha$ is a multiuser interference cancellation coefficient which takes values between 0 to 1 and quantifies the level of MUI. In particular, when the MUI is canceled perfectly, the SINR reverts to the SNR. On the other hand when $\alpha=1$, the MUI is fully present. In this paper, we assume that the value of $\alpha$ is fixed for convenience.

\section{Statistics of the SINRs of the Scheduled Users}

Starting from (1), the CDF of $\gamma_{S I N R_{m}}$, denoted by $P_{\gamma_{S I N R_{m}}}(\cdot)$, can be calculated in terms of the 2dimensional joint PDF of SNR of $m$-th desired scheduled user, $\gamma_{m: K}$, and the sum of the SNRs of the interfering $K_{s}-1$ scheduled users, $\sum_{\substack{n=1 \\ n \neq m}}^{K_{s}} \gamma_{n: K}$, denoted by $p_{\gamma_{m: K}, \sum_{\substack{n=1 \\ n \neq m}}^{K s} \gamma_{n: K}}(\cdot, \cdot)$ as

$$
P_{\gamma_{S I N R_{m}}}(\gamma)=\int_{0}^{\infty} \int_{0}^{\gamma(1+\alpha y)} p_{\gamma_{m: K}} \underset{\substack{n=1 \\ n \neq m}}{\sum_{s}} \gamma_{n: K}(x, y) d x d y
$$

After taking the derivative of (2) with respect to $\gamma$, the PDF of $\gamma_{S I N R_{m}}$, denoted by $p_{\gamma_{S I N R_{m}}}(\cdot)$ can be obtained as

$$
\begin{aligned}
p_{\gamma_{S I N R_{m}}}(\gamma) & =\frac{\partial}{\partial \gamma} P_{\gamma_{S I N R_{m}}}(\gamma) \\
& =\int_{0}^{\infty}(1+\alpha y) \cdot p_{\substack { \gamma_{m: K} \\
\begin{subarray}{c}{n=1 \\
n=m \\
n \neq m{ \gamma _ { m : K } \\
\begin{subarray} { c } { n = 1 \\
n = m \\
n \neq m } }\end{subarray}} \gamma_{n: K}(\gamma(1+\alpha y), y) d y
\end{aligned}
$$

Therfore, to obtain the statistics of $\gamma_{S I N R_{m}}$, we need to find the 2-dimensional joint PDF of $\gamma_{m: K}$ and $\sum_{\substack{n=1 \\ n \neq m}}^{K_{s}} \gamma_{n: K}$

3.1. Joint PDF of $\gamma_{m: K}$ and $\sum_{\substack{n=1 \\ n \neq m}}^{K_{s}} \gamma_{n: K}$

Applying our extended MGF-based approach we developed in [8], we accurately derive the joint PDF of $\gamma_{m: K}$ and $\sum_{\substack{n=1 \\ n \neq m}}^{K_{s}} \gamma_{n: K}$ in terms of its MGF and PDF. Because of space limitations, we only present the final results in this paper. The specific details behind the derivation of these results can be found in $[8,9]$.

Based on papers in $[8,9]$, we derive the generic expression of the joint PDF of $\gamma_{m: K}$ and $\sum_{\substack{n=1 \\ n \neq m}}^{K_{s}} \gamma_{n: K}$, by considering four cases i) $m=1$, ii) $1<m<K_{s}-1$, iii) $m=K_{s}-1$ and iv) $m=K_{s}$ separately. By considering these four cases, we can easily derive the target joint PDF as the expression given at the top of the next page.

Note that the operation in (4) involve only finite integrations of joint PDFs. Therefore, while a generic closed-form expression is not possible, the desired joint PDF can be easily numerically evaluated using standard mathematical packages such as Mathematica or Matlab and so on. 


\subsection{I.I.D Rayleigh Fading Special Case}

The generic non-closed form results presented in [8] apply to various fading environments. However for the i.i.d. Rayleigh fading environment both the joint MGF and PDF of interest can be found in closed-form expressions (i.e. expressions which do not involve any single integral). Using the classical inverse Laplace transform pair and property, we can obtain the target joint PDF over i.i.d. Rayleigh fading conditions in closed-form as

a. $m=1$ : Let $Z_{1}=\gamma_{1: K}, Z_{2}=\sum_{n=2}^{K_{s}-1} \gamma_{n: K}$, and $Z_{3}=\gamma_{K_{s}: K}$, then

$$
\begin{aligned}
p_{Z}\left(z_{1}, z_{2}, z_{3}\right)= & \frac{K !}{\left(K-K_{s}\right) !\left(K_{s}-2\right) !\left(K_{s}-3\right) ! \bar{\gamma}^{K_{s}}} \exp \left(-\frac{z_{1}+z_{2}+z_{3}}{\bar{\gamma}}\right) \\
& \times\left[1-\exp \left(-\frac{z_{3}}{\bar{\gamma}}\right)\right]^{K-K_{s}} U\left(z_{1}-z_{3}\right) \\
& \times \sum_{j=0}^{K_{s}-2}\left[(-1)^{j}\left(\begin{array}{c}
K_{s}-2 \\
j
\end{array}\right)\left[z_{2}-\left(K_{s}-2-j\right) z_{3}-j z_{1}\right]^{K_{s}-3}\right. \\
& \left.\times U\left(z_{2}-\left(K_{s}-2-j\right) z_{3}-j z_{1}\right)\right]
\end{aligned}
$$

b. $1<m<K_{s}-1$ : Let $Z_{1}=\sum_{n=1}^{m-1} \gamma_{n: K}, Z_{2}=\gamma_{m: K}, Z_{3}=$ $\sum_{n=m+1}^{K_{s}-1} \gamma_{n: K}$, and $Z_{4}=\gamma_{K_{s}: K}$, then

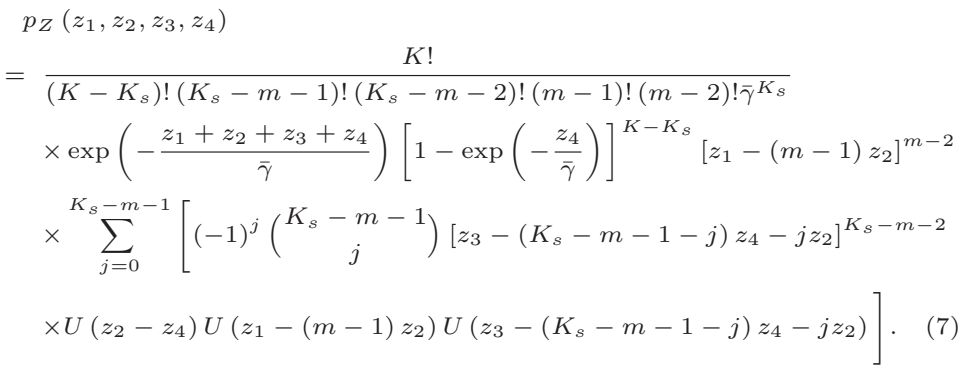

$$
\begin{aligned}
& \text { c. } \quad m=K_{s}-1: \text { Let } Z_{1}=\sum_{n=1}^{K_{s}-2} \gamma_{n: K}, Z_{2}=\gamma_{K_{s}-1: K} \text { and } \\
& \\
& Z_{3}=\gamma_{K_{s}: K} \text {, then } \\
& p_{Z}\left(z_{1}, z_{2}, z_{3}\right) \\
& =\frac{K !}{\left(K-K_{s}\right) !\left(K_{s}-2\right) !\left(K_{s}-3\right) ! \bar{\gamma}^{K_{s}}} \exp \left(-\frac{z_{1}+z_{2}+z_{3}}{\bar{\gamma}}\right) \\
& \quad \times\left[1-\exp \left(-\frac{z_{3}}{\bar{\gamma}}\right)\right]^{K-K_{s}} U\left(z_{2}-z_{3}\right)\left[z_{1}-\left(K_{s}-2\right) z_{2}\right]^{K_{s}-3} \\
& \quad \times U\left(z_{1}-\left(K_{s}-2\right) z_{2}\right) .
\end{aligned}
$$

d. $m=K_{s}$ : Let $Z_{1}=\gamma_{K_{s}: K}$ and $Z_{2}=\sum_{n=1}^{K_{s}-1} \gamma_{n: K}$, then

$$
\begin{aligned}
& p_{Z}\left(z_{1}, z_{2}\right) \\
= & \frac{K !}{\left(K-K_{s}\right) !\left(K_{s}-1\right) !\left(K_{s}-2\right) ! \bar{\gamma}^{K_{s}}} \exp \left(-\frac{z_{1}+z_{2}}{\bar{\gamma}}\right) \\
& \times\left[1-\exp \left(-\frac{z_{1}}{\bar{\gamma}}\right)\right]^{K-K_{s}}\left[z_{2}-\left(K_{s}-1\right) z_{1}\right]^{K_{s}-2} U\left(z_{2}-\left(K_{s}-1\right) z_{1}\right) \cdot(9)
\end{aligned}
$$

or equivalently

$$
\begin{aligned}
p_{Z}\left(z_{1}, z_{2}\right)= & \frac{K !}{\left(K-K_{s}\right) !\left(K_{s}-1\right) !\left(K_{s}-2\right) ! \bar{\gamma}^{K_{s}}} \exp \left(-\frac{z_{1}+z_{2}}{\bar{\gamma}}\right) \\
& \times\left[z_{2}-\left(K_{s}-1\right) z_{1}\right]^{K_{s}-2} U\left(z_{2}-\left(K_{s}-1\right) z_{1}\right) \\
& \times \sum_{j=0}^{K-K_{s}}(-1)^{j}\left(\begin{array}{c}
K-K_{s} \\
j
\end{array}\right)\left[\exp \left(-\frac{z_{1}}{\bar{\gamma}}\right)\right]^{j}
\end{aligned}
$$

Note that we have derived the joint PDF of $\gamma_{m: K}$ and $\sum_{\substack{n=1 \\ n \neq m}}^{K_{s}} \gamma_{n: K}$ only for $m=K_{s}$. For other cases, the marginals need to be computed in order to get the desired results. We can now apply these results to obtain the PDF of $\gamma_{S I N R_{m}}$ in (3) and therefore access the impact of the MUI on the performance of selection based parallel multiuser scheduling scheme.

\section{Performance Analysis}

In this section, we capitalize on the statistical results presented in the previous section to analyze the effect of interference on the throughput of the scheduled users assuming a selection based parallel multiuser scheduling scheme operating over i.i.d. Rayleigh fading conditions. In our simulation and numerical results, we use the selection based parallel multiuser scheduling scheme with $K=20$ and different values of $K_{s}$ and we assume i.i.d. Rayleigh fading conditions. To investigate the ASE, we also apply the conventional uncoded adaptive modulation of [10] with $N=8$. Based on page limitation, we only show some selected results only. However, you can find more detailed results from journal version.

\subsection{Total Average Sum Rate Capacity}

\subsubsection{Analysis}

The achievable rate, $R_{m}$ of the $m$-th scheduled user is limited by the individual instantaneous capacity $C_{m}$ as

$$
R_{m} \leq C_{m}=\log _{2}\left(1+\gamma_{S I N R_{m}}\right) .
$$

Therefore, the individual average sum rate capacity [11-13] can be obtained by averaging (11) with respect to $p_{\gamma_{S I N R_{m}}}(\gamma)$ as $\bar{C}_{m}=\int_{0}^{\infty} \log _{2}(1+\gamma) p_{\gamma_{S I N R_{m}}}(\gamma) d \gamma$. By adding the individual average sum rate capacities of the $K_{s}$ scheduled users, we can obtain the total average sum rate capacity as

$\bar{R}_{\text {total }} \leq \bar{C}_{\text {total }}=\sum_{m=1}^{K_{s}} \bar{C}_{m}=\sum_{m=1}^{K_{s}} \int_{0}^{\infty} \log _{2}(1+\gamma) p_{\gamma_{S I N R_{m}}}(\gamma) d \gamma$. 


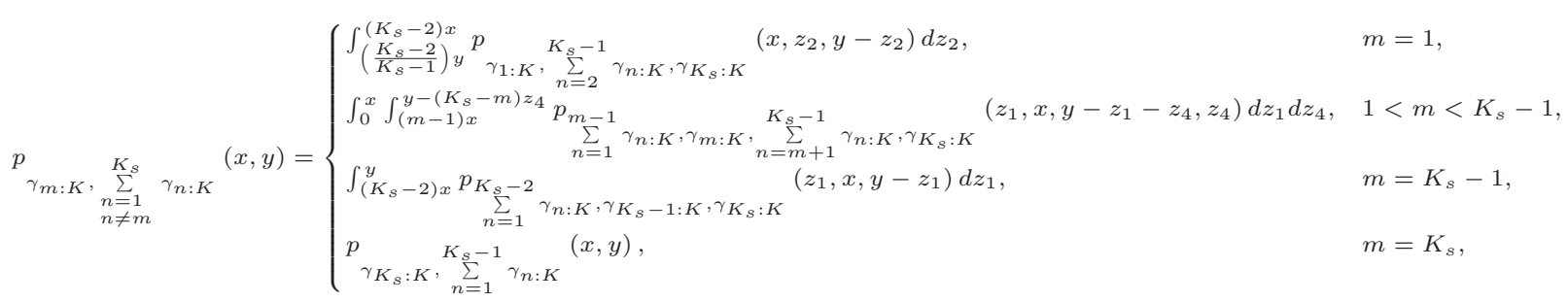

or equivalently

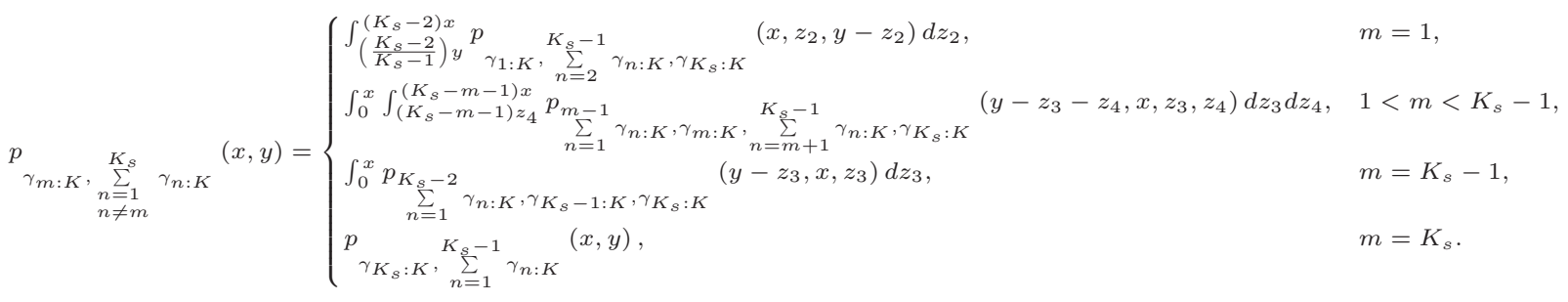

\subsubsection{Numerical Examples}

Fig. 1 show the total average sum rate capacity versus the number of scheduled users with various values of $\alpha$.

i) $\alpha=0$

In this case, the SINRs of the scheduled users are constant as $K_{s}$ increases. Therefore, the total average sum rate capacity is increasing continuously as $K_{s}$ increases. As a result, the system can schedule as many users as possible.

ii) $\alpha=1$

In the region of low $\bar{\gamma}$, the SINRs of the scheduled users are decreasing and finally saturates as $K_{s}$ increases but the values are almost constant. Therefore, the total average sum rate capacity is increasing as $K_{s}$ increases. As a result, in the region of low $\bar{\gamma}$, the system can schedule as many users as possible. In the region of high $\bar{\gamma}$, the SINRs of the scheduled users are decreasing considerably and finally saturated very quickly as $K_{s}$ increases. Therefore, the total average sum rate capacity is decreasing and finally saturates as $K_{s}$ increases. As a result, in the region of high $\bar{\gamma}$, there is no interest in scheduling multiple users simultaneously. If the fairness of access requires the simultaneous scheduling of multiple users in the region of high $\bar{\gamma}$, the system needs to select the number of scheduled users carefully by considering the total average sum rate capacity requirement and a channel status.

iii) $0<\alpha<1$

The SINRs of the scheduled users are decreasing as $K_{s}$ increases. This decrement is increasing as $\bar{\gamma}$ increases. The SINR curve as a function of $K_{s}$ for fixed value of $\bar{\gamma}$ approaches a straight line as $\alpha$ approaches 0 . In the region of low $\bar{\gamma}$, the SINR curve as a function of $K_{s}$ is almost straight regardless of the value of $\alpha$. Therefore, in the region of low $\bar{\gamma}$, the total average sum rate capacity is increasing as $K_{s}$ increases but for fixed value of $K_{s}$ the gain is decreasing as $\alpha$ increases. In the region of high $\bar{\gamma}$, in the case of very small $\alpha$, the total average sum rate capacity is just increasing but in the case of very large $\alpha$, the total average sum rate capacity is just decreasing as $K_{s}$ increases. Otherwise, the total average sum rate capacity is increasing as $K_{s}$ increases but the gain also depends on the value of $\alpha$.

Note that the total average sum rate capacity graph saturates more quickly and for fixed number of scheduled users, $K_{s}$, the gain is decreasing as $\alpha$ increases. Based on these facts, the system can schedule as many users as possible but the system needs to carefully select the number of scheduled users by considering the value of $\alpha$, the total average sum rate capacity requirement, and the channel status.

\subsection{Average Spectral Efficiency}

\subsubsection{Analysis}

The ASE of the $m$-th scheduled user is obtained as the sum of the spectral efficiencies $\left\{R_{n}\right\}_{n=1}^{N}$ of the individual codes, weighted by the probability $P_{n}$ that the SINR of this $m$-th scheduled user is assigned to the $n$-th region. Therefore, to obtain the overall ASE of the scheduled users for each time-slot, we just need to add the individual ASE of the scheduled users yielding

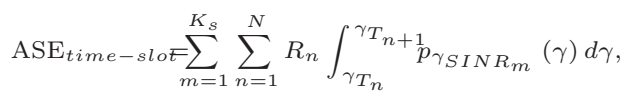


where

$$
\mathrm{ASE}_{m}=\sum_{n=1}^{N} R_{n} P_{n}=\sum_{n=1}^{N} R_{n} \int_{\gamma_{T_{n}}}^{\gamma_{T_{n+1}}} p_{\gamma_{S I N R_{m}}}(\gamma) d \gamma .
$$

\subsubsection{Numerical Examples}

In Fig. 2, we plot the ASE of the scheduled users as a function of the number of scheduled users with various values of $\alpha$ respectively. We can see from Fig. 2 that there exists a trade-off between the ASE and the number of scheduled users.

$$
\text { i) } \alpha=0
$$

In this case, the ASE does not depend on the type of adaptive modulation. The ASE always increases as $K_{s}$ increases. Therefore, the system can schedule as many users as possible.

ii) $\alpha=1$

When $\alpha=1$ and in the region of high $\bar{\gamma}$, the ASE decreases as $K_{s}$ increases. Therefore, in the region of high $\bar{\gamma}$, there is no need to schedule multiple users. In case that multiple users scheduling is needed in the region of high $\bar{\gamma}$, the system needs to select the number of scheduled users carefully by considering a system capacity requirement and a channel status. In the region of low $\bar{\gamma}$, the ASE of the scheduled users with conventional adaptive modulation $[10,14-19]$ is decreasing and finally becomes zero due to the discrete nature of adaptive modulation. Note that the SINRs of the scheduled users is decreasing and finally saturated as $K_{s}$ increases (but these values are almost constant although these values are changing) and almost of these values are very small compared with the minimum threshold of a conventional adaptive modulation. Therefore, for this case, the ASE is decreasing and finally becomes zero. However, if the system employs the appropriate type of adaptive modulation for low $\mathrm{SNR} \bar{\gamma}$, in the region of low $\bar{\gamma}$, the ASE may have a similar behavior as the total average sum rate capacity.

iii) $0<\alpha<1$

When $0<\alpha<1$, in the region of high $\bar{\gamma}$, the ASE with very small $\alpha$ is increasing but the ASE with very large $\alpha$ is decreasing as $K_{s}$ increases. Otherwise, the ASE is increasing and then decreasing and finally saturates as $K_{s}$ increases. The trade-off point is moving to left side as $\alpha$ increases. This means that when $\alpha$ is large, a trade-off happens for the smaller value of $K_{s}$.

Note that based on the theoretical background, in the region of low $\bar{\gamma}$, the ASE may have a similar behavior as the total average sum rate capacity. More specifically, in the region of low $\bar{\gamma}$, the ASE of the scheduled users may be increasing as $K_{s}$ increases. Therefore, in the region of low $\bar{\gamma}$, the system with the appropriate type of adaptive modulation for low SNR $\bar{\gamma}$ may schedule as many users as possible.
However, as you can see the results from Fig. 2, in the region of low $\bar{\gamma}$, the ASE of the scheduled users with conventional adaptive modulation with very large $\alpha$ is just decreasing. Otherwise, the ASE is increasing a little bit and then suddenly decreasing and finally saturates as $K_{s}$ increases due to the discrete nature of adaptive modulation.

Note that the ASE depends on both the value of $\alpha$ and the type of adaptive modulation especially in the low average SNR region. Therefore, if the system employs the appropriate type of adaptive modulation, in the region of low $\bar{\gamma}$, the system can schedule as many users as possible but in the region of high $\bar{\gamma}$, due to the trade-off between the ASE and the number of scheduled users, the system needs to select the number of scheduled users carefully by considering a system requirement and the channel status.

\section{References}

[1] R. Knopp and P. A. Humblet, "Information capacity and power control in single cell multiuser communications," in Proc. of IEEE Int. Conf. on Commun. (ICC'95), Seattle, WA, June 1995, pp. 331-335.

[2] G. B. Holter, M. -S. Alouini, G. E. Øien and H.-C. Yang, "Multiuser switched diversity transmission," in Proc. IEEE Vehicular Technology Conf. (VTC'O4-Fall), Los Angeles, CA, 2004, pp. 2038-2043.

[3] D. Gesbert and M. -S. Alouini, "How much feedback is multi-user diversity really worth?" in Proc. of IEEE Int. Conf. on Commun. (ICC'04), Paris, France, June 2004, pp. 234-238.

[4] Y. Ma and D. Zhang, "Performance of generalized selection multiuser scheduling over generalized fading channels," in Proc. IEEE Int. Wireless Commun. and Mobile Comput. Conf. (IWCMC'06), Vancouver, Canada, July 2006.

[5] S. S. Nam, M. -S. Alouini, K. A. Qaraqe and H.C. Yang, "Threshold based parallel multiuser scheduling," Proc. IEEE International Symposium on Personal, Indoor and Mobile Radio Communications (PIMRC'07), Athens, Greece, Sept. 2007.

[6] A. H. Nuttall, "An integral solution for the joint PDF of order statistics and residual sum," NUWC-NPT Technical Report, Oct. 2001.

[7] A. H. Nuttall, "Joint probability density function of selected order statistics and the sum of the remaining random variables," NUWC-NPT Technical Report, Jan. 2002.

[8] S.S. Nam, M. -S. Alouini, and H. -C. Yang, "An Unified Framework to determine the Joint Statistics of Ordered Statistics," IEEE Trans. Inform. Theory, submitted.

[9] — , "Impact of Interference on the Performance of Selection Based Parallel Multiuser Scheduling," IEEE Trans. Wireless Commun., submitted. 
[10] K. J. Hole, H. Holm, and G. E. Øien, "Adaptive multidimensional coded modulation on flat fading channels," IEEE J. Select. Areas Commun., vol. 18, no. 7, pp. 1153-1158, July 2000.

[11] M. Sharif and B. Hassibi, "On the capacity of MIMO broadcast channels with partial side information," IEEE Trans. Inform. Theory, vol. 51, no. 2, pp. 506-522, Feb. 2005.

[12] N. Jindal, "MIMO broadcast channels with finite rate feedback," IEEE Trans. Inform. Theory, vol. 52, no. 11, pp. 5054-5059, Nov. 2006.

[13] A. Goldsmith, Wireless Communications. New York, NY: Cambridge University Press, 2005.

[14] Y. -C. Ko, H. -C. Yang, and M. -S. Alouini, "Adaptive modulation and diversity combining based on output-threshold MRC," IEEE Trans. Wireless Commun., IEEE Trans. Wireless Commun., vol. 6, no. 10, pp. 3727-3737, Oct. 2007.

[15] M. -S. Alouini and A. Goldsmith, "Capacity of Rayleigh fading channels under different adaptive transmission and diversity techniques," IEEE Trans. Veh. Technol., vol. 48, no. 4, pp. 1165-1181, July 1999.

[16] A. J. Goldsmith and S. -G. Chua, "Variable-rate variable-power M-QAM for fading channels," IEEE Trans. Commun., vol. 45, no. 10, pp. 1218-1230, October 1997.

[17] M. S. Alouini and A. Goldsmith, "Adaptive modulation over Nakagami fading channels," Wireless Personal Communications, vol. 13, pp. 119-143, 2000.

[18] A. J. Goldsmith and S. -G. Chua, "Adaptive coded modulation for fading channels," IEEE Trans. Commun., vol. 46, no. 5, pp. 595-602, May 1998.

[19] Q. Liu, S. Zhou, and G. B. Giannakis, "Cross-layer combining of adaptive modulation and coding with truncated ARQ over wireless links," IEEE Trans. Wireless Commun., vol. 3, no. 5, pp. 1746-1755, Sept 2004.

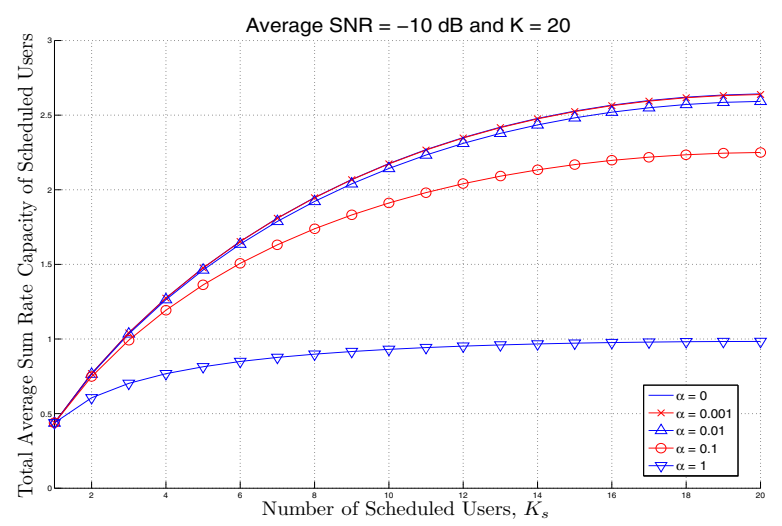

(a) Total average sum rate capacity for low average SNR $\bar{\gamma}=-10 \mathrm{~dB}$

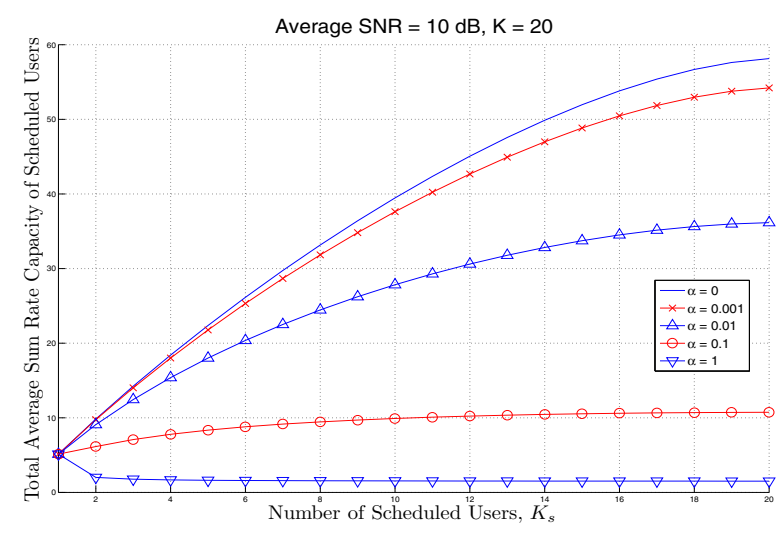

(b) Total average sum rate capacity for high average SNR $\bar{\gamma}=10 \mathrm{~dB}$

Figure 1: Total average sum rate capacity of scheduled users as a function of the number of scheduled users for selection based parallel multiuser scheduling over i.i.d. Rayleigh fading channels with $K=20$ and various values of $\alpha$.

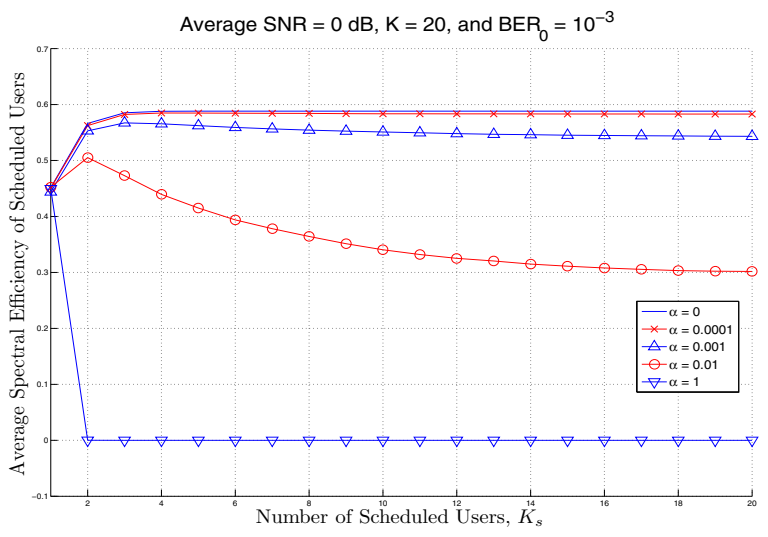

(a) ASE for low average SNR $\bar{\gamma}=-10 d B$

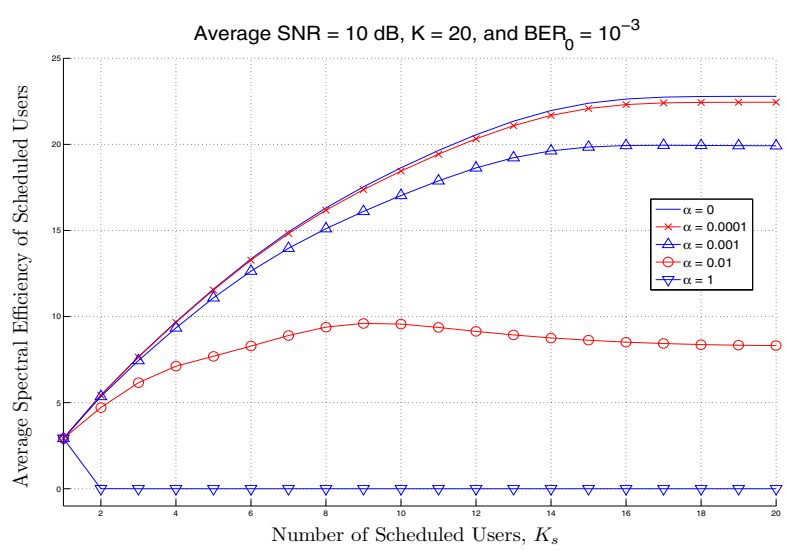

(b) ASE for high average SNR $\bar{\gamma}=10 d B$

Figure 2: ASE of scheduled users as a function of the number of scheduled users for selection based parallel multiuser scheduling over i.i.d. Rayleigh fading channels with $K=20$ and various values of $\alpha$. 